\title{
Textbook of Palliative Medicine
}

Bruera E, Higginson IJ, Ripamonti C, von Gunten C, editors. Hodder Arnold Company, London, United Kingdom, 2009. Softcover, 1095 pages. ISBN 978-0-340-96624-2. \$110.00

More than 170 palliative care experts from around the world joined forces in composing this textbook. It is indeed magnificent to have a concentrated pool of experts, from diverse geographic locations, providing a global perspective on the topic of palliative care. Most of the contributors are physicians, with a few representatives from nursing, social work, rehabilitation, epidemiology, and administration. Unfortunately, there is no representation from pharmacy, respiratory therapy, or nutrition.

The book has 16 sections covering a wide spectrum of topics, including the development of palliative medicine, bioethics, pain and other symptoms, emergencies in palliative medicine, and interdisciplinary issues. Each chapter is further divided into short sections. The book has some helpful features, such as a summary of key learning points and a complete list of references at the end of each chapter. For those who would like to further immerse themselves in a certain topic, symbols in the reference section identify seminal primary articles, key review papers, and management guidelines. Evidence scores on 3 levels are also provided: systematic review or meta-analysis, well-designed randomized controlled trial (RCT), and non-RCT or cohort study. Of special interest to pharmacists are the 14 chapters gathered under the topic of pain, with discussions on special populations such as pediatric patients, elderly patients, and patients with challenges related to addiction.

The downside of having numerous writers for a book such as this is that readers must switch among a collage of writing styles, which makes the book challenging to read at times. For 
instance, the chapter on spiritual care is the only one that uses case studies. Keeping the chapters brief and therefore easy to read has its advantages, but the resulting text sometimes lacks the comprehensive detail that readers may require. A couple of section headings are ambiguous: the section entitled "Assessment and Management of 'Other' Problems" discusses topics such as hydration, wound care, hope, and counselling, and "Specific Conditions and Situations" covers 13 topics that are mostly unrelated. This makes it difficult for the reader to retrieve the desired information without frequently consulting the index. Another disadvantage of having many contributors is that related information may be scattered and repeated in different chapters. Examples include physical medicine, which is covered in both chapters 15 and 16; episodic pain, which is embedded in a couple chapters within the section on pain; and pediatric concerns, which appear in the sections on pain and interdisciplinary issues. As well, the book could certainly have benefited from sections on psychosocial care, spirituality, and medication management; poor medication adherence in particular is a common problem in palliative care.

In summary, this textbook represents an effort to provide a vast amount of information on palliative medicine. It is successful in offering a global perspective, but for those looking for a "how to" text, this will not be the answer.

Ayesha Hassan, BScPharm, MSC

Pharmacy

Royal Inland Hospital

Kamloops, British Columbia 